\title{
Nonconventional Method of Polymer Patterning
}

\author{
Oleksiy Lyutakov, Jiri Tuma, Jakub Siegel, Ivan Huttel and Václav Švorčík
}

Additional information is available at the end of the chapter

http://dx.doi.org/10.5772/46143

\section{Introduction}

The unical properties of polymers, their cheap coast and possibility of easy chemical or physical modification, make these materials ideal building blocks for nano- or micropatterning. Techniques for polymers fabricating on nano- and micro-length scales span a wide range, from improved conventional lithographic methods to more recent materials and chemical advances that rely on self-organization of block copolymer. In addition to traditional methods, there are a number of techniques used exclusively in polymer materials processing. The most famous of them include molding, writing and printing, laser scanning, self-organization and surface instabilities utilization.

Nano-imprint lithography (NIL), first proposed in work [1], and represents a high resolution and high throughput polymer lithography technique. The general principle of NIL consists in mechanical deformation of the polymer layer with a stamp presenting a surface topography. Different modification of NIL includes ultraviolet or temperature assisted polymer curing, application of solvent vapor for material softening and so on. In a research laboratory, nano-imprint lithography works very well and the structure with nanometer scale can be prepared by this way. However, for industrial application, some problem must be solved. Main disadvantages of NIL can be attributed to flatness of stamp and substrate, local roughness of prepared patterns, stamp design issue, stamp contamination, and material shrinkage. Generally, nano-imprint lithography is still considered as a next generation lithography technique.

An alternative method introduced in work [2] has been proven to overcome most of drawbacks of traditional lithography. This method is named soft lithography and relies on using elastomeric polymers as soft molds to obtain patterned surfaces on other polymeric materials. The soft elastomeric stamp can be used either as a vehicle for molecular surface patterning (in the case of so-called micro-contact printing $(\mu \mathrm{CP})$ ) or to create three dimensional reliefs, as in micro-molding in capillaries, micro-transfer molding or solvent-assisted molding. 
Schematic representation of different variants the soft lithography process is given in the work [3]. Soft lithography technique was successfully applied in the fabrication of polymer patterns with dimensions down to the sub-100nm scale.

A direct-write assembly is a fabrication method that employs a controlled translation stage, which moves a pattern-generating device, for example, ink deposition nozzle, to create materials with controlled architecture and composition [4]. Arbitrary 3D structures can be prepared by this method. For the polymeric ink solidification occurs either upon deposition into a coagulating reservoir or UV exposure. This method can be characterized to be bioinspired because they copy a nature process - direct ink writing in the form of spider webs.

There are several processes which can play a role in polymer modification under laser scanning. Major processes occurring during polymer modification by excimer laser can be subdivided to: material transport under ablation threshold, ablation and polymer surface chemistry modification [5-7]. When polymer surfaces are exposed to a polarized pulsed laser to more than several hundred pulses with a sub-threshold flounce for ablation, ripple structures appear if the photons are absorbed with a high absorption coefficient in the surface layer only several hundred angstroms thick [5]. Possible explanation of observed phenomena consists in the materials transport under light interference pattern. The incident laser beam can interact with the reflected or scattered by surface inhomogeneities light and form the light interference pattern. Then the polymer flow occurs due to effects of temperature or electrical gradients. This method can be applied to the most of conventional polymers. The resulting periodicity is limited by the applied laser wavelength and by the complex response of the material to the laser illumination [7].

When polymer films are exposed to pulsed laser beams at a proper wavelength with beam energy above the ablation threshold energy, photoablation takes place [8]. In a low-fluence range an incubation period exists, i.e. after absorbing the laser energy the polymer starts to swell, and subsequently ablated materials are ejected. In a high-fluence range a shock wave front is formed and ablated material is ejected instantaneously with high speed. Usually the ablation of polymers includes depolymerisation of materials and evaporation of lowmolecular weight products $[9,10]$.

Another example of polymer modification by pulsed laser exposure is the surface chemistry modification [12.13]. For this purpose pulse fluencies of the UV laser should be higher than those used for the periodic ripple formation [10]. By exposing polymer surfaces to pulsed UV lasers, chemical properties of polymer surfaces can be improved to more desirable ones.

In our experiments we used the periodical laser scanning for polymer surface modification.

\section{Polymer modification by laser scanning}

At the first stage the optical properties of polymers were changed in desired way by suitable dotation. We used a porphyrine, or disperse red (DR1) as dopants with large absorption coefficients. 
Thin polymer films doped by organic dye were prepared by spin-coating technique. For the polymer dotation two ways were used. In the case of bulk dotation the solution of polymer was mixed with organic dye, dissolved in dichlorethane. In the case of DR1 the organic dye was added as dopant or chemically connected with polymer. Fig. 1 gives the chemical structure of used materials.

A

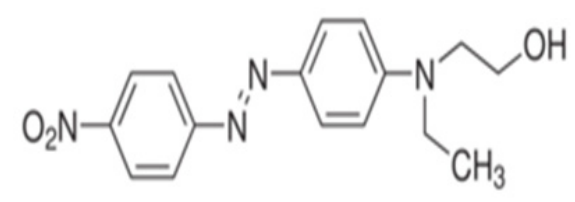

B

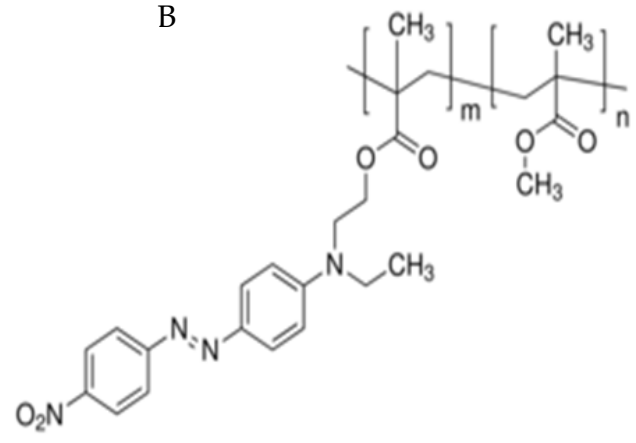

Figure 1. Chemical structure of Disperse Red 1 (A) and DR1 covalently linked to PMMA.

For surface dotatiton the vacuum deposition technique was applied. In this case the layer of pristine polymer was prepared by spin-coating and served as a substrate for next vacuum deposition of porphyrine. Vacuum deposition of porphyrine was performed under $10^{-6}$ Torr pressure and at $1 \mathrm{~A}$ electric current. For preparation of more complex structures the combination of both methods was used.

The doped polymer films were modified by laser scanning and simultaneous mechanical sample movement. This technique of polymer modification was recently proposed by our team [14]. Chosen polymer area is scanned by continual laser beam line by line. Laser beam was focused into spot with approximate diameter $0.5 \mu \mathrm{m}$. Laser operating at $405 \mathrm{~nm}$ wavelength and $0.1 \mathrm{~W}$ laser power was applied. The laser light of applied wavelength is expected to be absorbed by porphyrine molecules. Sample mechanical movement is added to the laser scanning. The velocity of the sample movement was $2 \mu \mathrm{m} / \mathrm{sec}$ [14]. The process is schematically depicted in Fig. 2.

For preparation of more complex structures both techniques of dotation were used. Firstly, polymer films were doped by bulk dotation and modified by laser scanning. Then, in a second step, the porphyrine was deposited on the surface of modified polymer, the sample was turned by $90^{\circ}$ regarding to previous orientation and scanned again. 


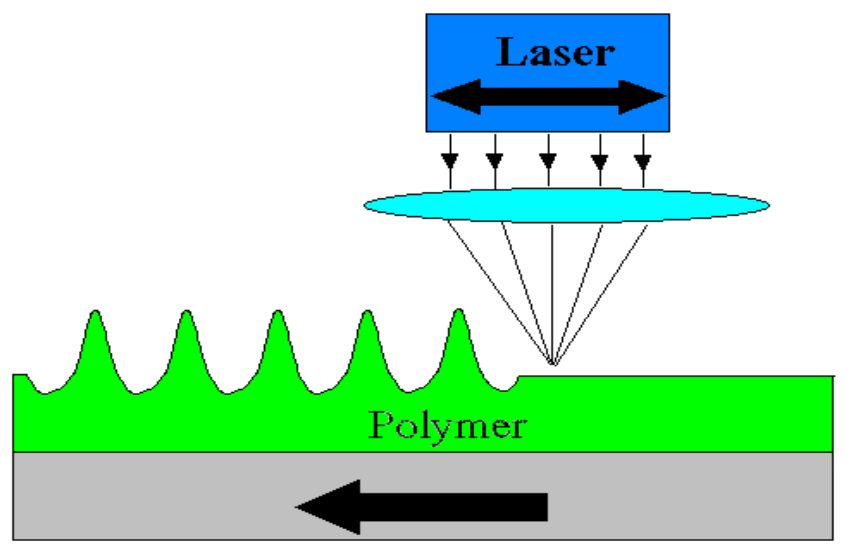

Figure 2. Schematic of the present experimental arrangement. Polymer is deposited by spin coating onto glass wafer, polymer surface layer is doped with porphyrin by deep coating. Then the surface is scanned by focused laser beam, together with simultaneous mechanical movement of polymer film. Direction of the mechanical movement is given by arrow.

Fig. 3 gives the typical structure prepared by laser scanning and simultaneous sample movement. Thin polymer film doped by porphyrine in bulk was exposed to focused beam of laser light with wavelength appropriate to maximum of porphyrine molecules absorption. Part A of the figure corresponds to application of laser scannning and gives the optical image and surface distortion at the boundary of exposed and measured by AFM. It is evident, that after the laser modification the polymer tends to form two protruding surface structures on the boundary of scanned area. It should be noted, that appearance of the structure occurs only in the direction of laser scanning (left and right sides of image). Formation of the structure in perpendicular direction (top and bottom sides of image) was never observed. It could be assumed, that polymer tends to flow in the direction of more pronounced temperature gradient. When the simultaneous mechanical movement of sample is added the periodical structure is formed (see Fig. 4B). Prepared structure exhibits the system of well ordered surface maximum and minimum peaks. In other words, the surface profile represents periodical array of lines along which the polymer mass was pulled above initial flat surface.

Surface patterning of pure polymer films was performed by covering the surface of polymer by suitable dye (porphyrine or phthalocyanine) and subsequent laser scanning. From the practical point of view it seems to be interesting to choose the polymer photoresist for patterning. The structure, prepared by laser scanning in our case or by another method can be strengthened and fixed by following UV light illumination. One of examples is the application of soft lithography techniques for patterning of commonly used photoresist Su-8 [15-17]. It must be noted, that in our case bulk dotation of Su-8 is not applicable, during the laser illumination two concurrent processes will occur - polymer flow and crosslinking. 
At the fist stage the pure Su-8 film was deposited onto Si substrate. In the next step, Su-8 layers covered by porphyrine were exposed to laser scanning with simultaneous sample movement. Fig. 4A shows the structure obtained after the first stage of patterning. It is evident, that prepared structure shows some tendency to be "periodical“, but the quality of this periodicity is "bad" (Fig. 4A). However, the next laser illumination results in material redistribution and sufficient improvement of the structure quality. The Fig. $6 \mathrm{~B}$ gives the surface profile of prepared structure after laser scanning and subsequent simple, homogenous laser illumination. The differences between Figs. 4A and B are evident, but the nature of observed phenomenon, the driving forces for material flow and structure improvements, are not clear now. In the next step prepared structures were fixed by UV light illumination and developed in dichlorethane. As can be expected, cross-linked Su-8 is not soluble and only top layers of porphyrine are dissolved. Typical surface profile of prepared structure is given in the Fig. $4 \mathrm{C}$.
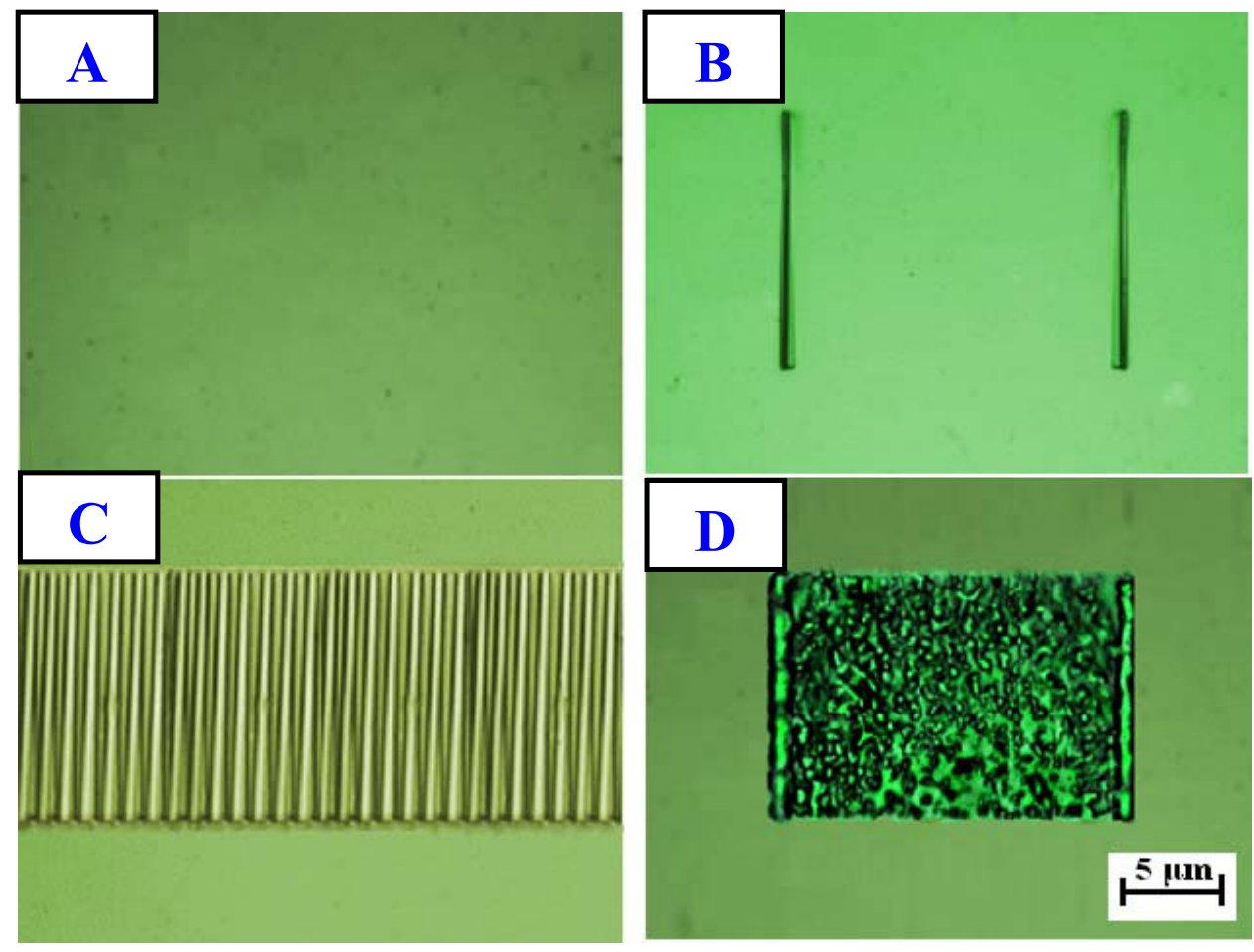

Figure 3. Various stages of the surface patterning as a function of the laser intensity and the velocity of the mechanical movement. Laser scanning has been applied accross $20 \times 20 \mu \mathrm{m}$ area at the centre of the image. Part A - correspond to laser intensity of $0.03 \mathrm{~mW}, \mathrm{~B}-0.12 \mathrm{~mW}, \mathrm{C}-0.12 \mathrm{~mW}$ and simultaneous mechanical movement, and $\mathrm{D}-0.25 \mathrm{~mW}$. 


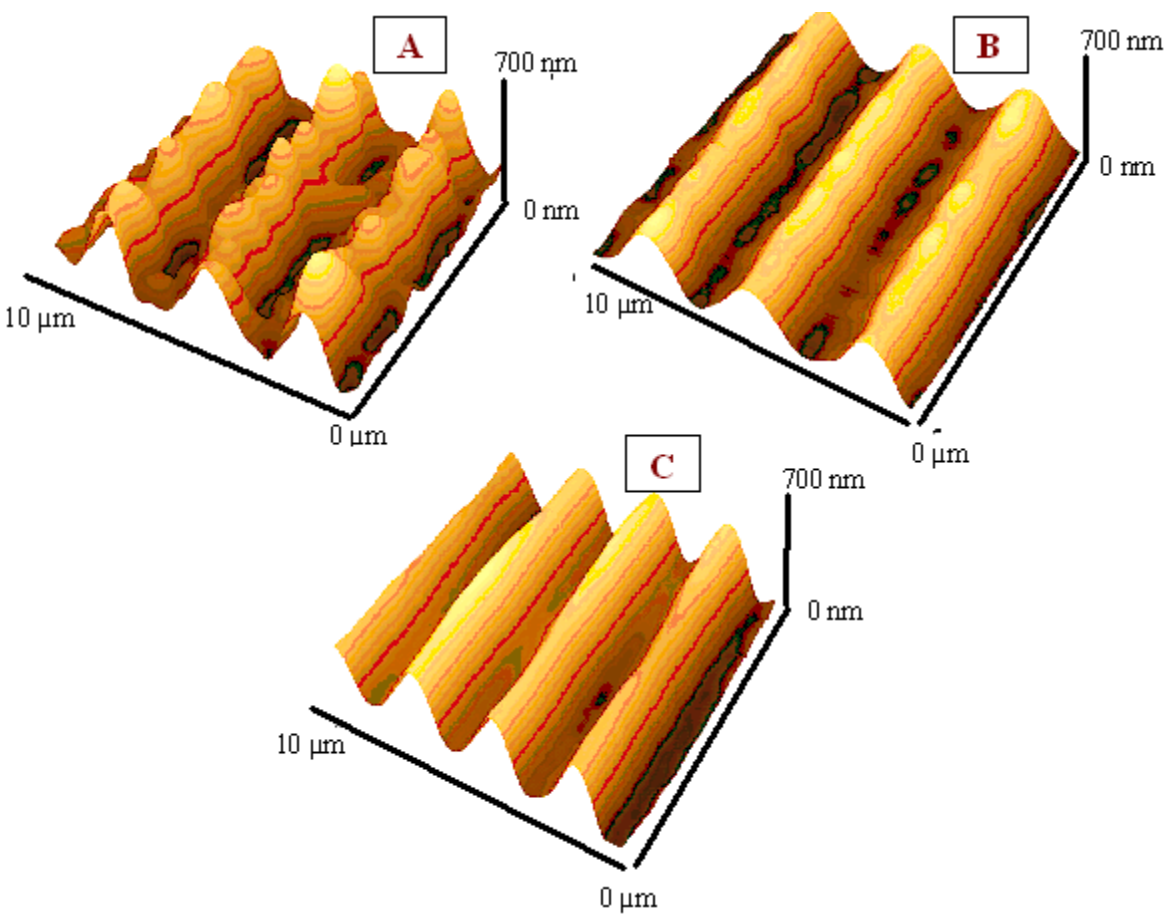

Figure 4. AFM images of surface of Su-8 with doped with porphyrine after modification by laser scanning and simultaneous mechanical movement: A- surface after application of laser scanning and sample movement, B- surface after subsequent laser illumination and C- surface of modified film exposed to UV light and treated in dichlorethane.

Proposed technique opens-up a possibility to utilize wider range of materials as dopant, regardless of their solubility. Class of phthalocyanines, affined with porphyrines, seems to be very interesting from the sensor application [18] point of view, however it is difficult to dissolve phthalocyanine in common solvents. Application of high vacuum deposition can help to avoid bad solubility of phthalocyanine. Different dyes, deposited on the top of polymer can serve as light absorber for patterning process and as "active“ material in next utilization of prepared structures e.g. in sensor application. In these case properties of polymer matrix remain invariable; which may be important is some specific applications. Additionally, evaporation of dyes onto formerly patterned polymer surface opens-up new way for more complex 2D polymer surface patterning.

Another technique of polymer dotation consists in chemical bounding of dye to polymer chain. Chemical structure of used molecules is given on the Fig. 1. Two sets of experiments were performed in this case. At first case the dye was added as dopant and in second case covalently bounded. The results of surface measurement by AFM are given on the Fig. 5. It is evident, that covalently bounding results in sufficiently highest amplitude of prepared structure. Additionally, this technique of polymer dotation opens a way for process reversibility. 


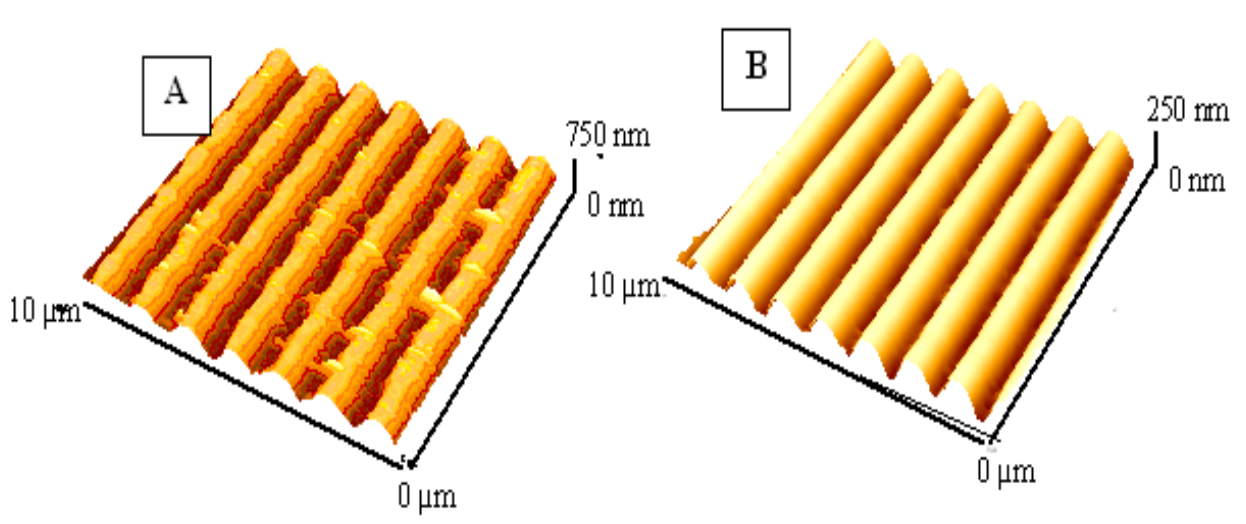

Figure 5. Surface structures of PMMAcoDR1 (A) and PMMA doped by DR1 (B). Surfaces were modified under laser intensity of $0.03 \mathrm{~mW}$ and $2 \mu \mathrm{m} / \mathrm{sec}$ speed of simultaneous mechanical movement.

\subsection{Mechanism of polymer flow}

Proposed mechanism of the structure formativ consists in polymer mass re-distribution governed by surface tension gradient (so-called Marangoni effect [19]). The classical mechanism of the Marangoni effect expressed in the formation of surface structures at the interface of two phases is the nonuniformity of surface tension due to temperature gradient [20]. In our experiments absorption of light during the scanning generates spatial variability of surface tension, usually responsible for Marangoni effect. To our opinion in the first step the polymer matrix is molted. At the next step periodical temperature gradient and the surface tensions gradient are created. As can be predicted by Marangoni phenomenon the polymer tends to flow from the region with smaller surface tension to the region with higher surface tension. On the boundaries of scanned region the polymer flow must be stopped because the materials outside of this region are not in the molten state. When continual mechanical movement of sample is added to the laser scanning, processes of polymer melting, flow and stopping achieves equilibrium and 2D periodical structure is created. The driving force for the structure formation is the magnitude of thermal gradient and related inhomogeneity in the surface tension. The initiation and propagation of this process at "stationary conditions" is well described in several works [31,32] from the theoretical point of view. Nevertheless, in the present case we deal with nonequilibrium conditions because of continuous sample movement.

\subsection{Surface chemistry}

For verification of surface or bulk chemistry changes occurring during laser irradiation the UV-VIS, IR and XPS analysis were performed. Fig. 6 gives the UV-VIS optical spectra obtained before and after laser patterning. Peak at the $405 \mathrm{~nm}$ corresponds to so-called 
Sorett band. From the figure is evident, that porphyrine molecules retain their configuration during laser modification.

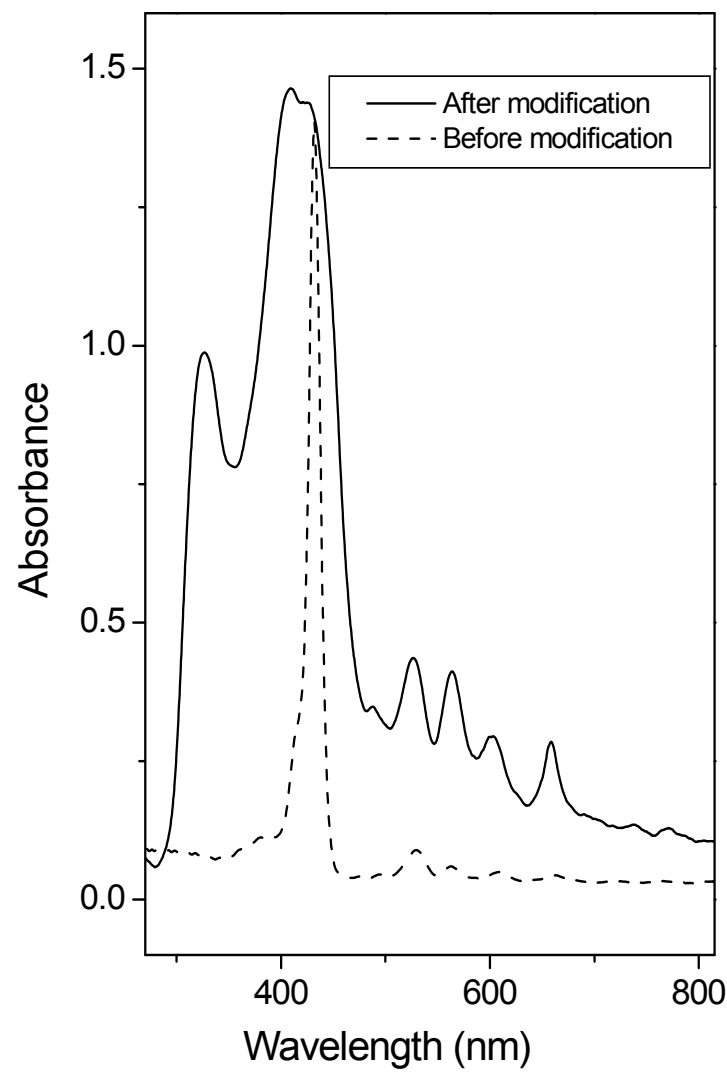

Figure 6. Absorption spectra of PMMA film doped by porphyrine before and after laser modification.

Surface chemistry of modified sample was measured by XPS analysis. Formation of surface patterns can arise not only due to the local heating of the polymer surface, but also due to local heat release in chemical and physico-chemical processes in the laser irradiated polymer. XPS was performed on PMMA samples doped on surface and in bulk. Relative element concentrations before and after surface modification are given in Table 1 . In the case of bulk doped PMMA the slight decrease in $\mathrm{C}$ concentration and increase in $\mathrm{N}$ and $\mathrm{O}$ concentrations were found. This result indicates absence of sufficient changes in polymer surface chemistry. Observed changes in element concentrations can be attributed to redistribution of polymer and dopant molecules in fused state. In the case of surface doped PMMA the considerably smaller concentration of $\mathrm{O}$ on the pristine surface was found, in 
comparison with that doted in bulk. This result could be expected, because the top layer of porphyrine screens the polymer molecules. Similarly, as well as in the case of bulk doted samples only minor alterations in surface chemistry after the laser modification were found. Small increase in $\mathrm{C}$ concentration and small decrease in $\mathrm{N}$ concentration can be explained in the terms of polymer flow and mixing of porphyrine/polymer layer.

\begin{tabular}{lccc}
\hline & \multicolumn{3}{c}{ Element concentration (in at. \%) } \\
\cline { 2 - 4 } & $\mathbf{C}(1 \mathrm{~s})$ & $\mathbf{O}(\mathbf{1 s})$ & $\mathbf{N}(\mathbf{1 s})$ \\
\hline Bulk doped PMMA & & & \\
before patterning & 64.3 & 24.8 & 10.9 \\
after patterning & 60.8 & 26.0 & 13.2 \\
\hline Surface doped PMMA & & & \\
before patterning & 83.8 & 5.6 & 10.6 \\
after patterning & 85.7 & 6.7 & 7.6 \\
\hline
\end{tabular}

Table 1. Surface concentration of elements $(\mathrm{C}(1 \mathrm{~s}), \mathrm{O}(1 \mathrm{~s})$ and $\mathrm{N}(1 \mathrm{~s}))$ in bulk and surface doped PMMA before and after the laser modification determined from XPS analysis.

Since the chemical changes of the polymer surface initiated by the laser irradiation are not substantive one can conclude that the surface patterning is mostly due to classical temperature effects.

Standard procedure for theoretical analysis of surface pattern in thin polymer films under temperature gradient is linear stability analysis $[22,23]$ according which the formation of surface structures is governed by the surface tension and material redistribution ratio due to heat transfer, where the temperature gradient once again destabilizes the situation, while the viscous drag damps disturbances. There are several cases, which can lead to periodical structure appearance under temperature gradient: (i) nonuniform density distribution over the layer of viscous liquid heated from one side so-called gravitational instability [35], (ii) so-called concentration instability appears in multi-component system due to difference in component concentration [22] and (iii) surface tension instability corresponds to differences in surface tension of homogeneous thin film due to temperature gradient, presented in these films [23].

In case of gravitational instability, stability of thin films limit is determined by the value of the Rayleigh dimensionless criterion and doesn't depend on the forces of surface tension. Rayleigh dimensionless criterion is strongly related to thickness of treated film. For verification of this mechanism the series of samples with different layer thickness were prepared and examined in the same way. Dependences of amplitude of prepared periodical structure on the sample thickness are given in Fig 3. In Fig. 3 the dependence for PMMA with two different molecular weights is shown. Samples were prepared with the same porphyrine concentrations and modified at the same laser light intensity. From Fig. 3 it is evident, that the amplitude of the patterns increases linearly with increasing sample thickness up to the thickness of about $2100 \mathrm{~nm}$ in both investigated cases. Above this threshold thickness the increasing rate slows down considerably. Appearance of threshold 
thickness and linear character of amplitude increase leads us to the conclusion that the polymer flow is not governed by gravitational instability. Rising part of curves can be explained by the amount of absorbed light energy increasing with the film thickness. The upper part of the curves can be attributed to limited light penetration - absorption in polymer when only thin surface layer of the polymer is affected.

Surface instability may also be attributed to nonuniform concentrations in multi-component system. In our experiments, where we used ether the pristine films or films with homogeneous concentration of dopant, this mechanism can be excluded.

Another reason for outbreak of surface instability may be the presence of surface tension gradients due to nonuniform temperature field of a system on the upper free surface. This mechanism seems to be more reasonable in the present case. In this case the structure formation must depend on the surface tension and viscous damp. With the aim to investigate the viscous damp influence, two PMMA with different molecular weights were examined since it is well known, that viscous damp of polymer is strongly related to their molecular weight. Linear polymer with the larger molecular weight will have greater value of viscous damp, than its low-molecular homolog. In our work we investigated PMMA with $1500 \mathrm{~K}$ and $15 \mathrm{~K}$ molecular weights. Sufficiently sharper structure with higher amplitude was formed in the case of PMMA with lower molecular weight. One can say, that utilizing of polymers with different molecular weight can result in differences in surface tension too. It should, however, be noted that surface tension of thin films is much more dependent on the chemistry of macromolecules rather than on molecular weight [23].

\section{Application}

\subsection{Optical components}

Periodical or ordered structures can serve as different optical components. In our works we examined the polymer lattice prepared onto polymer surface as light diffraction elements of waveguide coupler.

At the first stage the diffraction of light through the lattice was estimated. Because the spot of applied laser possesses the sizes of an order of square millimeters, system of lattices deposited abreast was prepared. Optical image of prepared structures is given in Fig. 7 . Considering difficulties in precise positioning of each lattice, some disagreement in their mutual orientation is observed. All lattices had identical periodicity, however were displaced relative to each other on some small step. For the next experiments the areas of $1 \times 1 \mathrm{~cm} 2$ were patterned on the sample surface. In the next stage the diffraction intensity of light passing through prepared structures were observed and photographed. Obtained image is shown in Fig. 8. From the Fig. 8 it is evident, that prepared structure exhibits satisfactory diffractive properties. However, some illegibility and vagueness in diffraction intensity maxima is observed. This discrepancy in diffraction can be attributed to disagreement in mutual ordering of both lattices. 

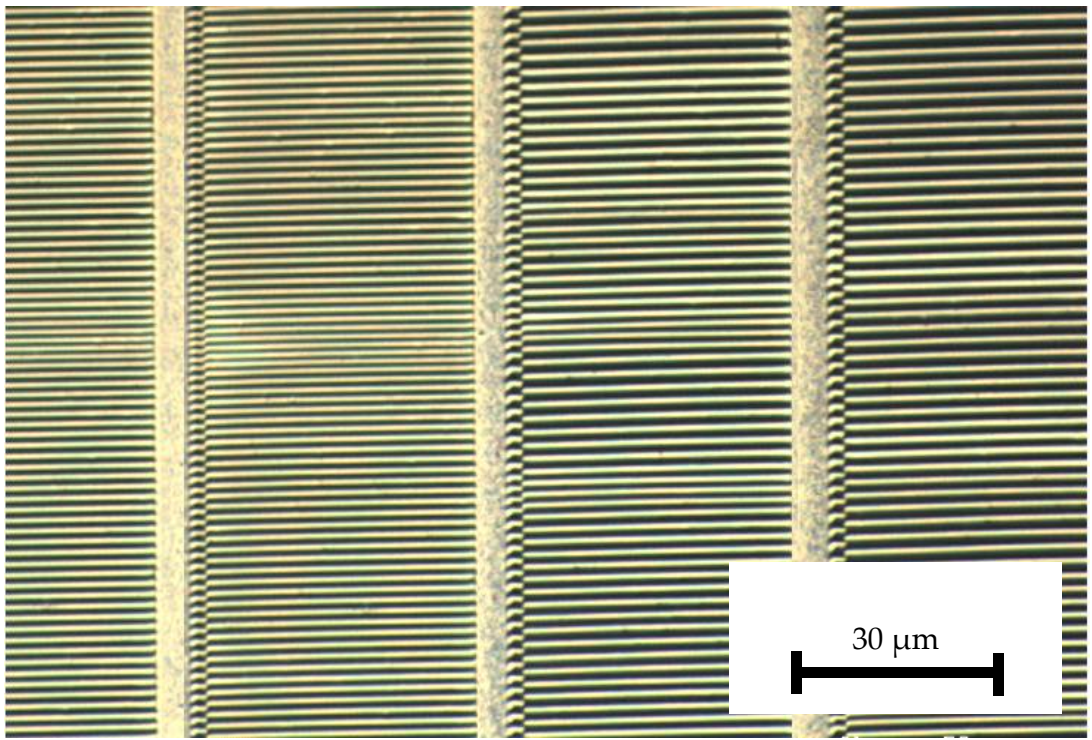

Figure 7. Optical image of system of lattices, prepared abreast on PMMA doped by $3 \%$ porphyrine.

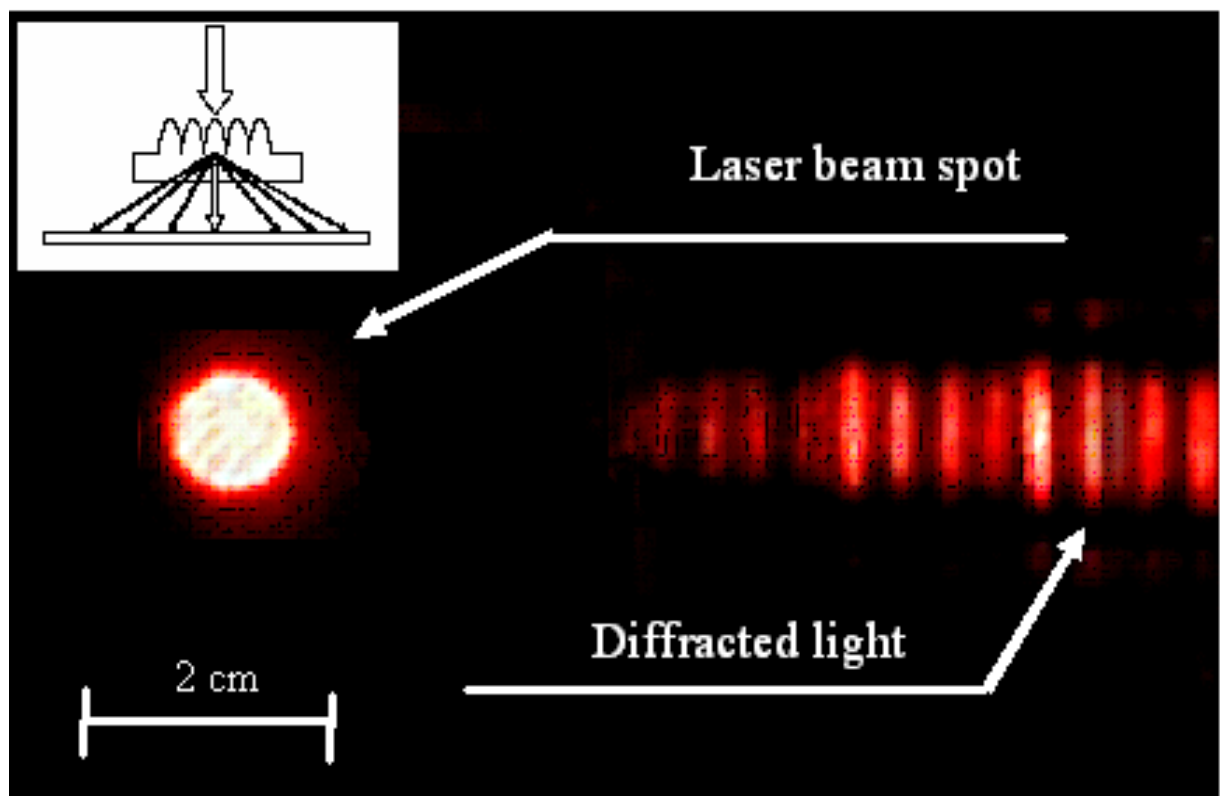

Figure 8. Diffraction pattern of laser light $(633 \mathrm{~nm})$ passed through a system of lattices deposited abreast on PMMA (1500k) doped by $3 \%$ porphyrine. Presented process is schematically depicted in the inset. 
One of the possible applications of prepared structure is coupling of light into waveguide. Example of utilization of prepared structure as waveguide coupler is given in Fig. 9. As well as in previous experiment the sample with system of lattices was used. In this experiment the sample was deposited onto rotation stage and the optimal angle of laser beam incident was founded. This angle corresponds to the most effective light coupling into planar waveguide. From Fig. 9 it is apparent, that a part of laser energy was tapered by lattices and travelled along a waveguide. The prepared profile of a surface pattern had sinusoidal character. From literature it is well known, that for light coupling the rectangular structure profile is more effective [24]. However, for laboratory conditions the light coupling exibits sufficient efficiency. Additionally, presence of good diffractive optical properties of prepared structures opens-up a way for their utilization in sensing and plasmonics.

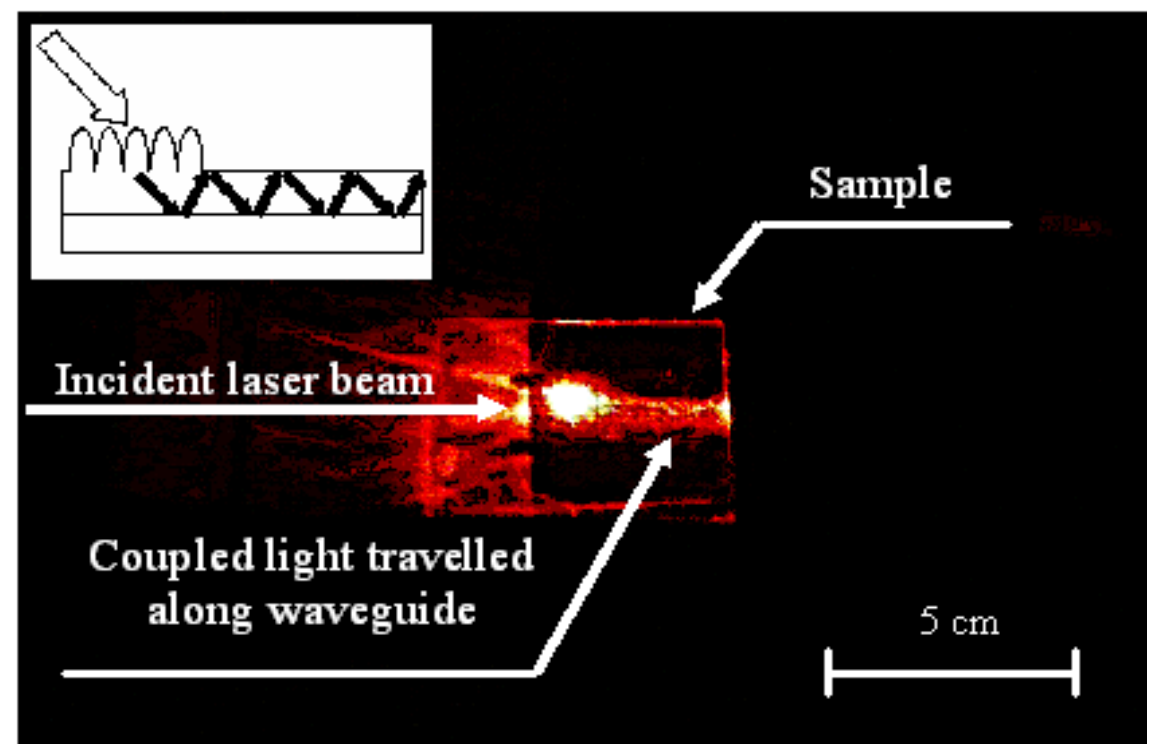

Figure 9. Coupling of light in planar waveguid through system of lattices deposited abreast on PMMA (1500k) doped by $3 \%$ porphyrine. Presented process is schematical depicted in the inset.

\subsection{Surface wettability}

Wetting phenomena on structured solid surfaces are of both fundamental and technological interest. The enhancement of hydrophobicity by roughness is described by either Wenzel or Cassie model, which includes water or air trapped in the structure below the drop. Engineered surfaces exhibiting superhydrophobicity or superhydrophility have been reported [24]. Periodical ordered surface patterning can be used for preparation of surface with anisotropic wetting behavior. This anisotropic wetting behaviour has been observed on one-dimensional (1D) patterned surfaces achieved either through chemical patterning or surface roughness. Surfaces with controlled anisotropic wetting, confining liquid flow to a single direction, have potential applications in microfluidic devices, evaporation-driven 
formation of patterns, and easy-clean coatings. A relatively strong anisotropic wettability was measured on imprinted hierarchical structures wchich combines micro and nanosized ordering of surface. Weak anisotropic wetting characteristics were observed on periodic polymer structures.

Some results of our work in this field is summarized in the table 2 Results is presented in the form of difference in contact angle of water and glycerol, when observation is performed from two perpendicular point of view.

As was reported in the literature, the drop contact anle can be a function of drop volume. The phenomenon of dependence of wettability on the drop evaporation time is given, for example in the work [25]. From this reason in the next step the wettability of surface for "small" volume of deposited drop was studied. Glycerol was chooses as probe liquid due to hear great sensitivity and lowest evaporation time. Because of experimental restriction we can't direct measure the contact angle and demonstrate the view from the top from optical microscope. Fig. 10 gives the behavior of glycerol droop deposited onto substrate patterned substrate. The anisotropy in drop behavior is apparent. From figure can be concluded, that substrate are sufficiently more wettable in the direction paralel to prepared lattice pattern..

\begin{tabular}{|c|c|c|c|c|}
\hline Sample & Amplitude $(\mathbf{n m})$ & Periodicity $(\boldsymbol{\mu m})$ & \multicolumn{2}{|c|}{ Difference in contact angle } \\
\hline- & - & - & Glycerol & Water \\
\hline 1 & 100 & 1.35 & 0.8 & 0.2 \\
\hline 2 & 300 & 1.35 & 2.5 & 0.6 \\
\hline 3 & 300 & 3.5 & 7.5 & 6.5 \\
\hline
\end{tabular}

Table 2. Anisotropic wetting of modified PMMA surface.

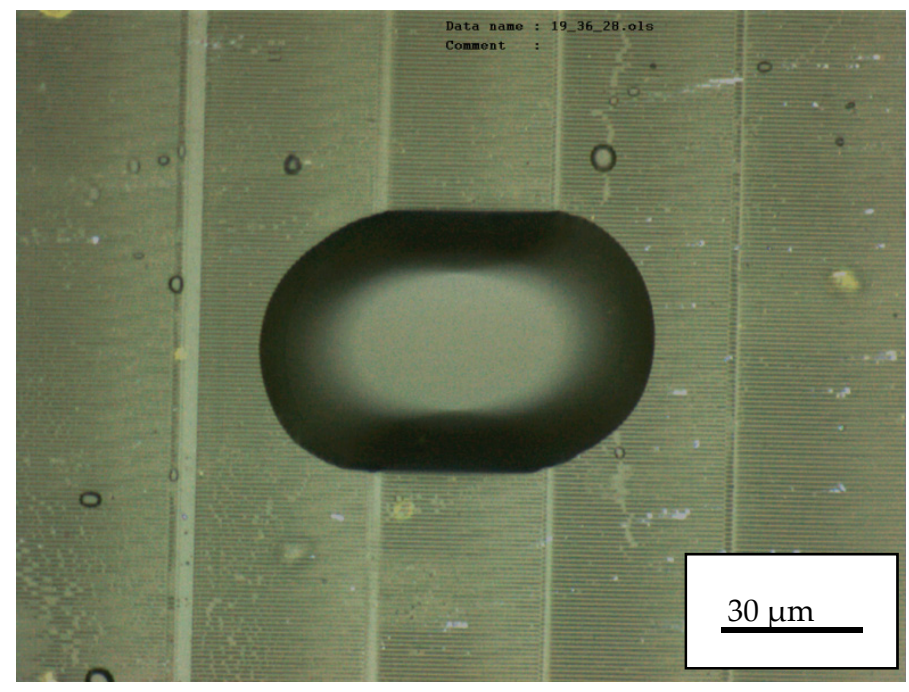

Figure 10. Glycerol drop deposited onto modified PMMA surface. 


\subsection{Surface coating}

Ordered array of hybrid polymer-metal structures can found their application in areas such as plasmonics [26], new photonics metamaterials [27], sensing [28] and catalysts [29]. Large electromagnetic field enhancements near anisotropic nanoparticles make metal nanowires attractive candidates as substrates for surface-enhanced Raman scattering [30]. Observed colossal increase in the effective scattering cross section of a molecule deposited on nanostructure metal surface (by 4-6 orders of magnitude) can lead to single-molecule detection. Additionally, various types of near field optical lithography utilizing enhancement of optical field make use of different types of ordered metal structures. Light interaction with specially designed metallic structures results in effects unattainable with naturaly occurring materials, e.g. negative permeability [30], negative refractive index [28] and nonlinear effects in magnetic meta-materials [29]. These observations led to a new area of photonics called optical meta-materials that have been exponentially growing over the last few years.

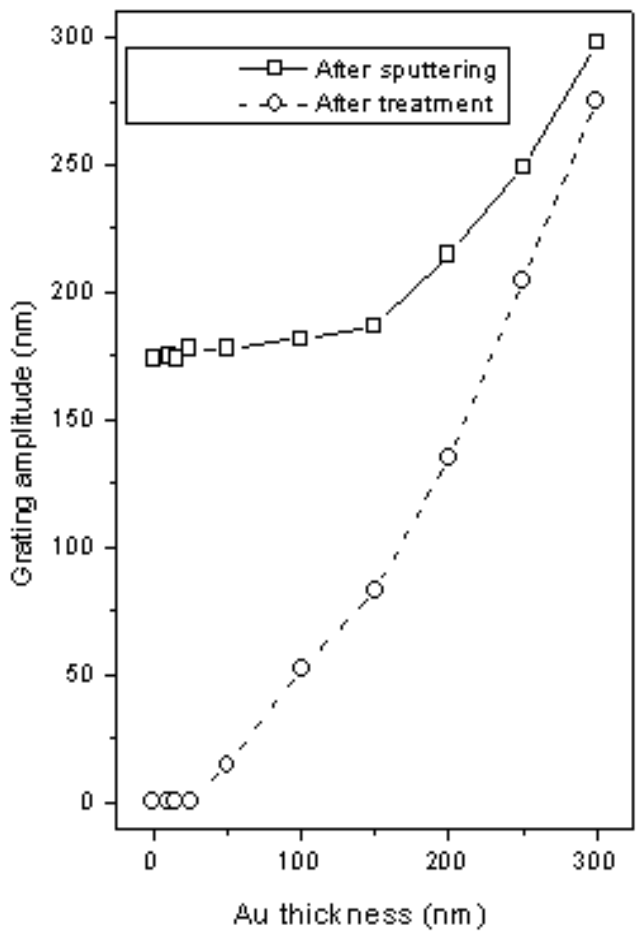

Figure 11. Change of grating amplitude during the Au deposition and thermal annealing.

Examination of surface coating of structure prepared by two different methods was performed. The metals [Ag and Ga] were deposited by sputtering technique. Additionally, it is well known, that annealing of thin metal layer can results in material rearrangement and formation of nano- and micro-clusters. Dependences of amplitude of prepared structure on the deposited thickness of metal and subsequent thermal treatment are given in Fig. 11 and 
Fig.12. From figures is evident, that in both case amplitude of structures tends to slightlly increase during metal deposition. Possible explanation consists in primary deposition of metal on the top of sinusoidal pattern due to shadow effect. Subsequent annealing, however leads to fundamentally different results in the case of Au and Ga coating. Results of thermal treatment at $200^{\circ}$ during 10 hours for coated polymer surface are given in Figs. 13 and 14 .

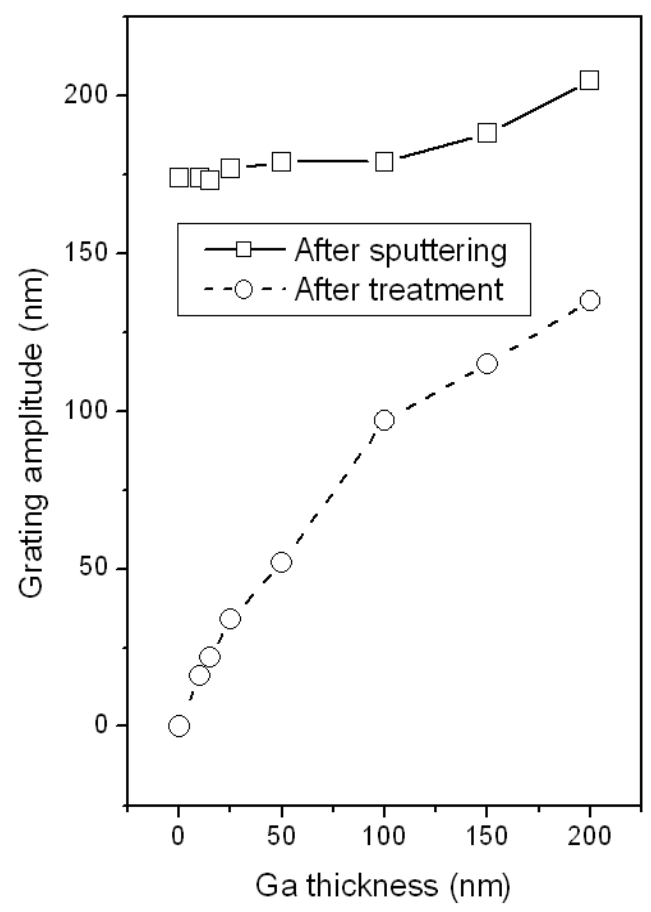

Figure 12. Change of grating amplitude during the Ga deposition and subsequent thermal annealing.
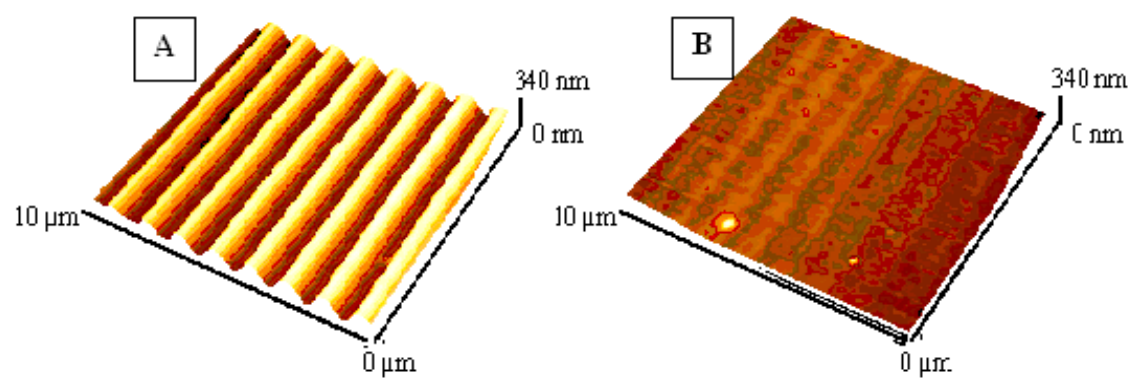

Figure 13. Thermal treatment of patterned PMMA surface covered by $50 \mathrm{~nm}$ Au layer. Part A polymer surface before treatment. Part B - after treatment 


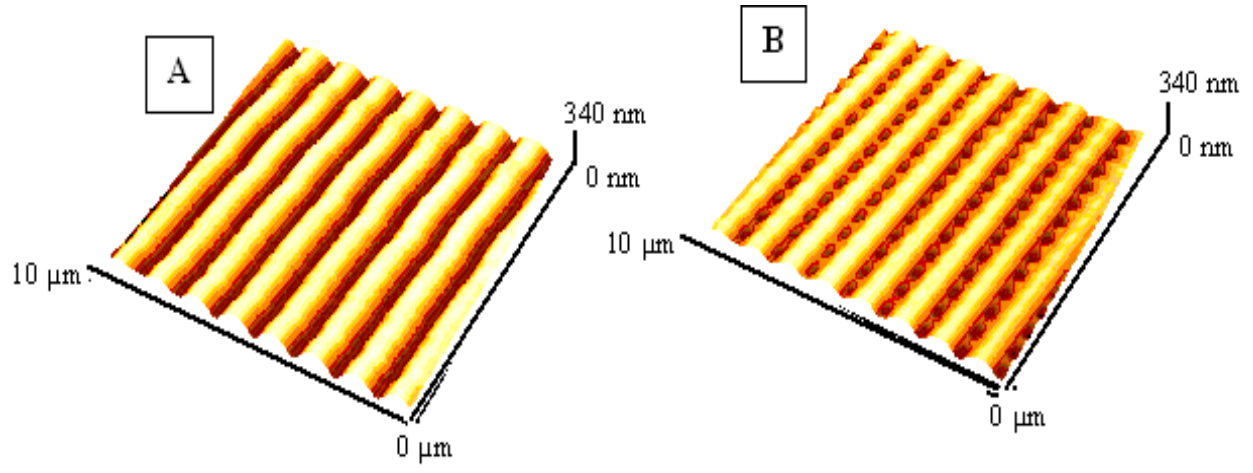

Figure 14. Thermal treatment of patterned PMMA surface covered by $50 \mathrm{~nm}$ Ga layer. Part A - polymer surface before treatment. Part B - after treatment

Additionally, changes in optical properties of doped polymer were observed. Fig. 15 shows the transmission spectra of PMMA doped by porphyrine before and after thermal annealing. Possible explanation of observed phenomenon consists in dopant molecule migration and selforganization into crystal structure.

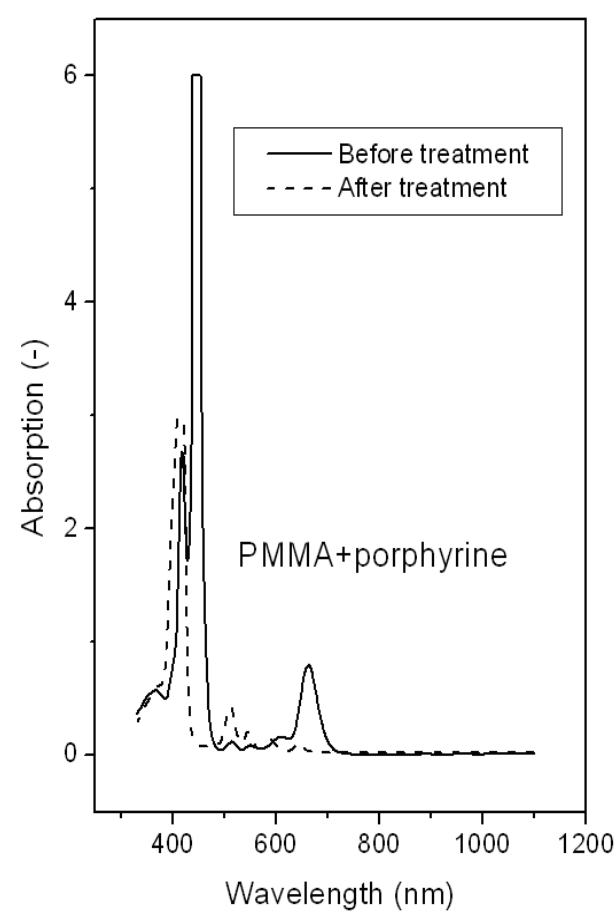

Figure 15. Optical absorption of PMMA film doped by 3\% porphyrine before and after thermal treatment at $200 \mathrm{o}$ C. 


\section{Thin film instability enhanced by external electric field}

One relatively new, promising technique for polymer patterning is a process based on hydrodynamic instabilities of liquid polymer surface induced by external electric field [31, 32]. Recently thin film instability was reported which could be used for creation of wellcontrolled patterns on thin polymer films [33,34]. This technique allows creation, with a high accuracy and reproducibility, of well defined polymeric patterns with dimensions ranging from a few tens of $\mathrm{nm}$ to a few $\mathrm{mm}$. A lot of experimental work was done by Russel et al. and by Chou et al. [31,32]. By technique proposed by Chou and called LISC (lithography induced self-construction) remarkable uniformity can be obtained over a large surface, but inevitable mechanical contact between polymer and mask introduces an additional degree of roughness to the waveguides which adds a significant scattering loss contribution to the overall waveguide loss. Another mechanism of polymer flow under external electric field leads to appearance of ordered non-continuous structure. In the work [32] this mechanism was called LISA - lithography induced self-assembling.

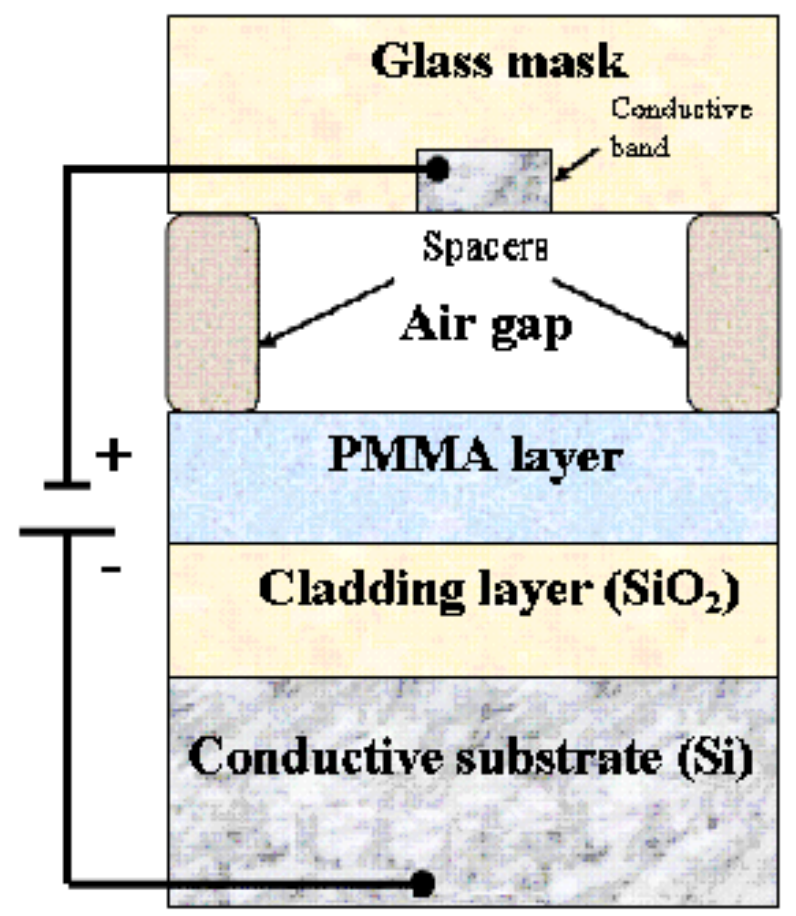

Figure 16. Scheme of polymer surface modification. Electric field effect the heated PMMA layer in the space between conductive silicon substrate with a $\mathrm{SiO}_{2}$ layer and a metallic (chrome) stripe $50 \mu \mathrm{m}$ wide on a glass lithographic mask in distance $25 \mu \mathrm{m}$ from the PMMA layer.

The present experiments were performed on polymethylmethacrylate (PMMA) and novolak resin (Su-8). Experimental set-up is schematically given on Fig. 16. Polymer films were 
sandwiched between conductive substrate and glass mask with conductive strip $(50 \mu \mathrm{m}$ wide and few $\mathrm{mm}$ long). The part of the sample was exposed to electric field created between strip electrode and Si backing. The $25 \mu \mathrm{m}$ long distance from the upper electrode to the polymer film was choose and the field intensities of electric field do not exceed a breakdown limit of about $5 \cdot 10^{6} \mathrm{~V} / \mathrm{m}$. The whole assembly under the electric field was heated in an oven to temperature $160^{\circ} \mathrm{C}$ for Su- 8 and $275^{\circ} \mathrm{C}$ for PMMA films. After exposure, the sample was allowed to cool down spontaneously to room temperature under the continued effect of the electric field. In the case of Su-8 films were subsequently irradiated with UV light.

Fig. 17 represents the dynamism of channel growth in the case of PMMA. The polymer layers were exposed to the field under increased temperature for various periods of time. The homogeneity of the prepared structures depended on the period of the electric field effect. From figure is obvious that the prepared structure were discontinuous and inhomogeneous until 60 minutes of field effect. In figure there is also visible that the channel growth starts at "random" spots along the full length of the future channel. These discontinuities get connected after a longer effect of the electric field (under increased temperature). Continuous and homogeneous structure appears after 60 minutes.
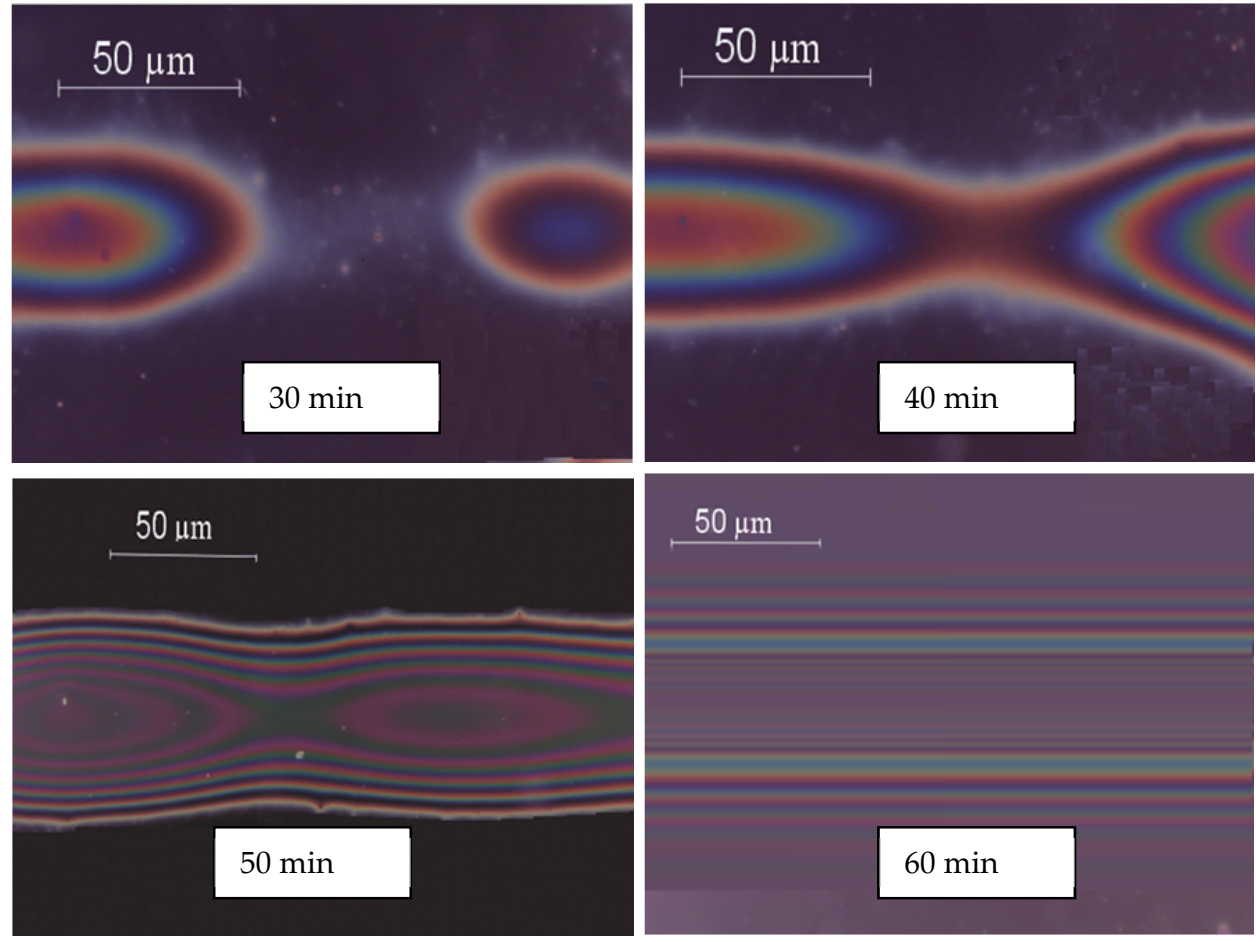

Figure 17. Photo of PMMA structures prepared under the electric field $\left(2.5 \cdot 10^{6} \mathrm{~V} / \mathrm{m}\right.$, at $\left.275 \pm 5^{\circ} \mathrm{C}\right)$ for various time (min), from optical microscope. 
A typical profile of the surface of the structure prepared by heating the entire surface and under local effect of the electric field, as measured perpendicular to the structure, is presented in Fig. 18. We can see that a redistribution of material occurs during the interaction of the electric field with dielectric material and a part of the polymer layer is pulled out in the direction given by the electric field vector. Fig. 18 shows that "pulling" the polymer layer in the area exposed to the electric field makes the layer around to the prepared structure thinner. Fig. 18 also presents the dependence of height of the prepared structures on applied electric field intensity. The thickness of the prepared structures increased with the intensity of the electric field, as we expected. Higher intensity of the electric field results in an increased electrostatic force and subsequent elongation of the structure along the direction electric field. The prepared channels were homogeneous and continuous.

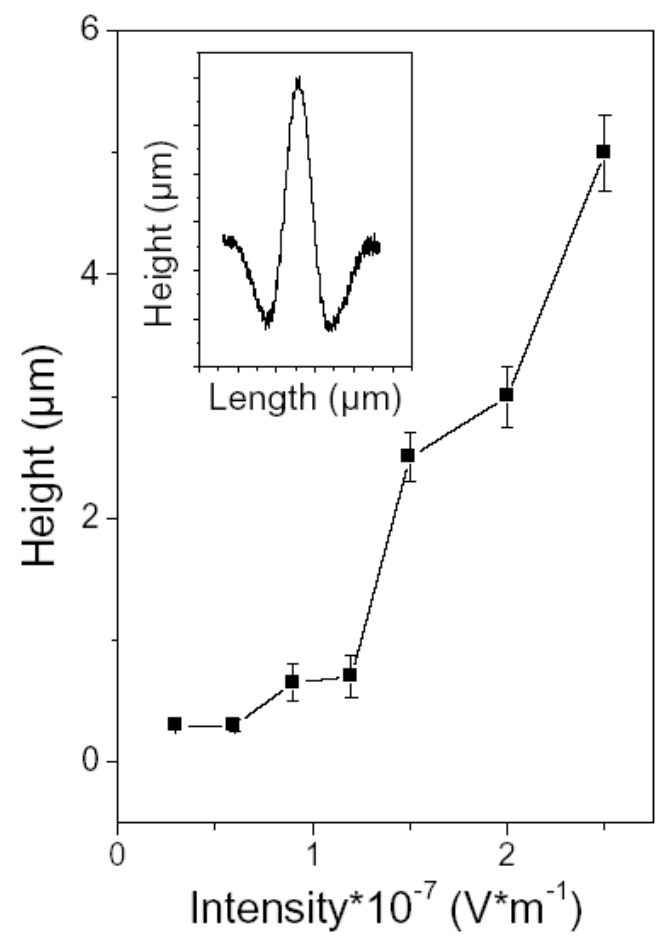

Figure 18. Dependence of height of structures prepared under temperature $275 \pm 5^{\circ} \mathrm{C}$ on an applied electric field. The insert shows the typical profile of a channel prepared under $1.2 \cdot 106 \mathrm{~V} / \mathrm{m}$.

Fundamentally different results were obtained in the case of Su-8 growing. Fig. 19 represents two series of experiments performed at two electric field intensities of 5.0 and $2.5 \times 10^{6} \mathrm{~V} / \mathrm{m}$ and with exposure times ranging from 3-15 min. For short exposures longitudinal homogeneous channel structures are formed. For longer exposures the homogenous structures disappear and system of dots is created. It may therefore be 
concluded that LISC mechanism prevails in initial stages of the pattern formation which is replaced by LISA mechanism at longer exposures. The LISC to LISA transition comes earlier for higher field intensity.

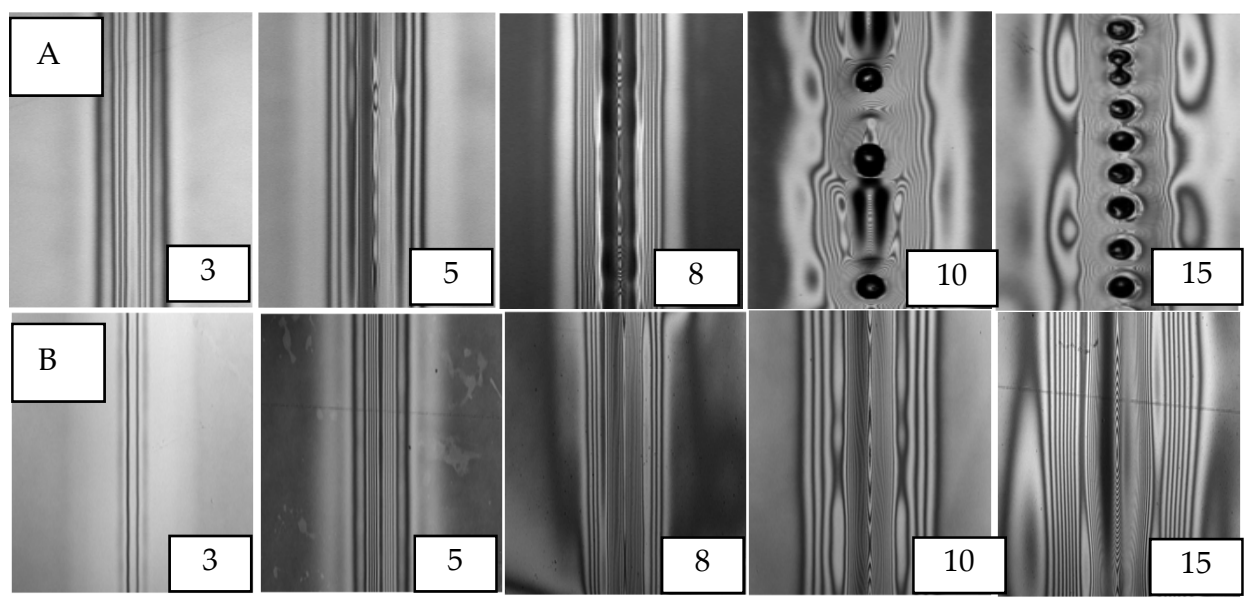

Figure 19. Confocal microscope images illustrating the evolution of the structures in dependence on the exposure time in $\mathrm{s}$. The numbers are the exposure times in minutes. The structures were created under two electric field intensities of $5 \times 10^{6}(\mathrm{~A})$ and $2.5 \times 10^{6} \mathrm{~V} / \mathrm{m}(\mathrm{B})$ and with the initial distances between the mask and the polymer surface of $40(\mathrm{~A})$ and $5 \mu \mathrm{m}$ (B), respectively.

\section{Application}

The above described technique seems to be prospective for fabrication of polymer based optical devices. The method is simple, free of disadvantages of conventional techniques. It gives highly reproducible results and is suitable for production of high-quality polymer waveguides. The above described LISC technique can be applied for preparation of optical channel waveguide. Waveguiding structure was prepared under following conditions: electric field intensity of $2.5 \times 0^{6} \mathrm{~V} / \mathrm{m}$, substrate temperature of $160^{\circ} \mathrm{C}$, distance between mask and polymer $50 \mu \mathrm{m}$, exposure time $5 \mathrm{~min}$. After pattern formation sample was irradiated by UV light with the aim to improve mechanical properties and stability of the polymer structures. Upon exposure to UV radiation, the oligomer systems form highly cross-linked networks, which exhibit low intrinsic absorption in the wavelength range extending from 400-1600 nm. The longitudinal parameters of the created linear structure were controlled by profilometry and the height and width of the structure were found to vary within $\pm 5 \%$ along the whole structure length. Within these limits the produced structures can be considered as homogeneous and continuous. The refractive index profile in a cut of the typical rib waveguide structure is shown in Fig. 20A. It is evident, that the technique enables simple fabrication of rib-type waveguide avoiding main disadvantages of traditional photolithography. Wave-guiding properties of the structure were examined by feeding it 
with $632.8 \mathrm{~nm}$ laser light. Distribution of light intensity radiated from the end of channel waveguide was measured and is given in the Fig. 20B as function of space coordinates.

Previous results were used for computer simulation of optical properties of the fabricated structures. By substitution to the RSoft software of measured polymer refractive index and the structure surface profile the inside and output optical field distribution were calculated using beam propagation method. The calculated output optical field is given in Fig 20C. In addition, calculation proved that the effective refractive index for fundamental mode is approximately 1.6276 at $632.8 \mathrm{~nm}$. The prepared multimode structure supported 30 modes at wavelength $632.8 \mathrm{~nm}$.
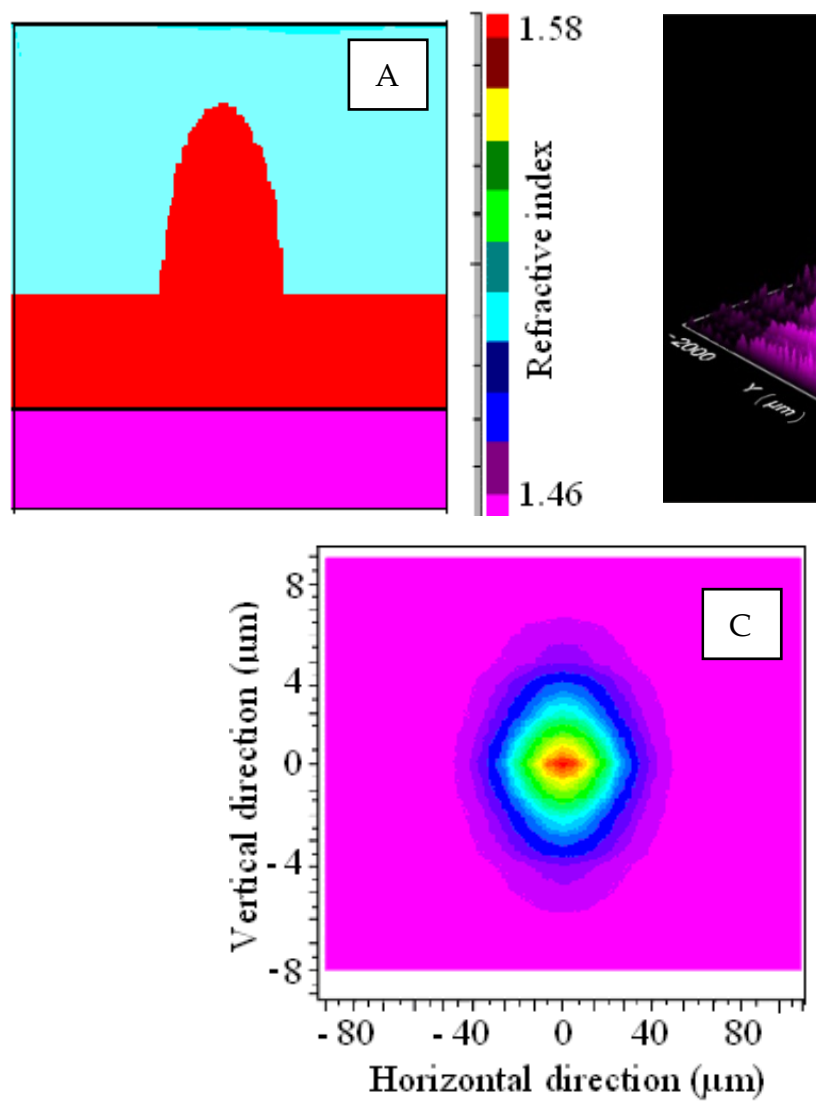

Figure 20. (A) Cross-section refractive index profile for typical rib waveguide, (B) output optical field $(632 \mathrm{~nm})$.distribution radiate from the end of rib channel waveguide, $(C)$ optical far field distribution for rib channel waveguide calculated in RSOFT software. 
Example of prepared Y-splitter of waveguide power is given on Fig. 21. Part A represents the microscopy image of applied top mask. Part B show the microscope image of prepared structure. Part $C$ gives the light throughput prepared structure. It is well visible, that light are guided by prepared structure and divided into two beams according to structure geometry.
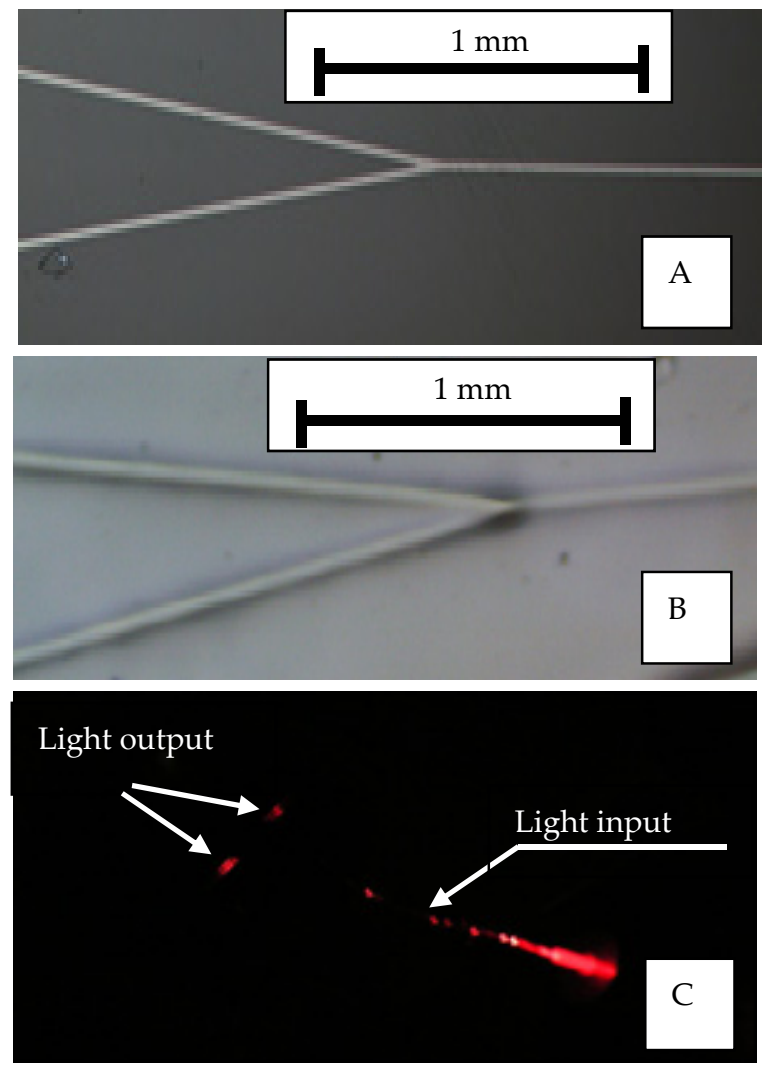

Figure 21. Junction of waveguide power. Part A represents the microscope image of applied top mask Part $B$ show the microscope image of prepared structure. Part $C$ give the light througput prepared structure.

\section{Conclusions}

In summary, this chapter gives a comprehensive insight into the problematic of examined new method for polymer surface patterning. For surface modification we used PMMA with different molecular weight and polymer photoresist. Mechanism of structure creation was analyzed and found to be dependent on surface tension gradient introduced by laser light 
scanning. Applications of prepared structures in photonics as diffraction elements or waveguide coupler are also given.

There is a considerable technological significance in the experiments we performed. It was shown that a nontactile nonablative method without wet chemistry is able to create surface structures of interesting properties. The polymer patterning step can take place in ambient air and can be strongly modulated by change of speed of polymer sample movement, molecular weight of polymer and dopant concentration.

Another described method of polymer surface modification consists in application of thin film instability enhanced by external electric field. Linear structures were created by the effect of locally limited electric field in thin polymer films heated to fluid temperature. The form and the shape of the structures were examined as a function of the exposure time, electric field intensity and the initial distance between mask and the film. It was shown that both continuous or dot structures can be created in dependence on the process parameters. For short processing times continuous structures are produced and with increasing times the continuous structures disintegrate into a system of dots. With increasing intensity of the electric field the height of the structures increases. The technique was successfully applied for preparation rib, channel waveguides. Optical properties of prepared light-guiding structures were measured and calculated using beam propagation method.

The above described technique seems to be prospective for fabrication of polymer based optical devices. The method is simple, free of disadvantages of conventional techniques. It gives highly reproducible results and is suitable for production of high-quality polymer waveguides. However, it should be noted, that the response to external electric field is not the same for different polymers. Apparently the quality and the shape of the structures created by the effect of the external electric field depend on the polymer type, its molecular weight, temperature of flow, molecular structure ect. Therefore the conditions of pattern preparation must be optimalized for each particular case.

In addition, surface coating of patterned polymer by thin metal layer were also described. Thermal treatment of prepared structure results in pattern disappearance or conservation of surface pattern, depending on the thickness and properties of metal coating.

\section{Author details}

Oleksiy Lyutakov, Jiri Tuma, Jakub Siegel, Ivan Huttel and Václav Švorčík

Department of Solid State Engineering, Institute of Chemical Technology, Prague, Czech Republic

\section{Acknowledgement}

This work was supported by the GA CR under the projects 108/11/P840, 108/11/P337, and 108/12/1168 and MPO CR under the project FR-TI3/797. 


\section{References}

[1] Chou SY, Krauss PR, Renstrom PJ (1995) Appl. Phys. Lett., 67: 3114-3117

[2] Kumar A, Whitesides GM (1993) Appl. Phys. Lett., 63: 2002-2004.

[3] Quake SR, Scherer A (2000) Science, 290: 1536-1538.

[4] Lewis JA, Gratson GM (2004), Mater. Today, 7: 32-47.

[5] Gratson GM, Xu MJ, Lewis JA (2004) Nature, 428: 386-388.

[6] Yablonovitch E, (1987) Phys. Rev. Lett., 58: 2059-2061.

[7] Jin, HJ, Kaplan DL, (2003) Nature, 424: 1057-1060.

[8] Yokoyama S, Nakahama T, Miki H, Mashiko S (2003) Thin Solid Films, 15: 438-439,

[9] Li CF, Dong XZ, Jin F, Jin W, Chen WQ, Zhao ZS, Duan XM (2007) Appl. Phys. A, 89: 145-148.

[10] Klein S, Barsella A, Leblond H, Bulou H, Fort A, Andraud C, Lemercier G, Mulatier JC, Dorkenoo K (2005) Appl. Phys. Lett., 86: 211118-211120.

[11] Sherwood T, Young AC, Takayesu J, Jen AKY, Dalton LR, Chen AT, (2005) IEEE Phot. Tech. Lett., 17: 2107-2109.

[12] Guo R, Xiao SZ, Zhai XM, Li JW, Xia AD, Huang WH (2006) Opt. Express, 14: 810-816.

[13] Serbin J, Ovsianikov A, Chichkov B (2004) Opt. Express, 12: 5221-5228.

[14] Lyutakov O, Huttel I, Siegel J, Svorcik V (2009) Appl. Phys. Lett. 95: 17-19.

[15] Ho K, Tsou Y, (2001) Sensors Actuat. B 77: 253-256.

[16] Melloni A, Martinelli M (2002), J. Lightwave Techn. 20: 296-299.

[17] Lyutakov O, Tuma J, Prajzler V, Huttel I, Hnatowicz V, Svorcik V (2010) Thin Solid Films 519: 452-456

[18] Kafesaki M, Tsiapa I, Katsarakis N, Koschny T, Soukoulis CM, Economou EN (2007) Phys. Rev. B 75: 235114-235117.

[19] Malkin AY (2008) Colloid J. 70: 673-676..

[20] Bestehorn M, Pototsky A, Thiele U (2003) Europ. Phys. J. 33: 457-461.

[21] Reichenbach J, Linde H (1981) J. Colloid Interface Sci. 84, 433-432.

[22] Perez-Garsia C, Carneiro G (1991) Phys. Fluids A 3: 292-297.

[23] Ashley KM, Raghavan D, Douglas JF, Karim A (2005) Langmuir 21: 9518-9521.

[24] Pollock C, Lipson M (2003) Integrated Photonics, Kluwer Academic Publishers, Boston.

[25] Erbil HY, Meric RA (1997) J. Phys. Chem., 101: 6867-6873.

[26] Murphy C, Gole A, Hunyadi S, Orendorff C (2006) Inorg. Chem., 45:7544-7547.

[27] Nie SM, Emery SR, Science 1997, 275:1102-1108.

[28] Zhou J, Zhang L, Tuttle G, Koschny T, Soukoulis C (2006) Phys Rev B, 73:041101-041105.

[29] Zhang S, Fan WJ, Panoiu NC, Malloy KJ, Osgood RM, Brueck SRJ (2005) Phys. Rev. Lett., 95:137404-137407.

[30] Linden S, Enkrich C, Dolling G, Klein M, Zhou J, Koschny T, Soukoulis C, Burger S, Schmidt F, Wegener M Europ. Phys. J. (2006) 12:1097-1101.

[31] Schaffer E., Thurn-Albrecht T, Russel T, Steiner U (2000) Nature 403: 874-877.

[32] Schaffer E, Thurn-Albrecht T, Russel T, Steiner U (2001) Eur. Phys. Lett. 53: 518-521.

[33] Verma R, Sharma A, Kargupta K, Bhaumik J (2005) Langmuir 21: 3710-3712.

[34] Shankar V, Sharma A (2004) J. Colloid. Interf. Sci. 274: 294-298. 